\title{
THE ULTRA-VIOLET AND VISIBLE ABSORPTION SPECTRA OF PHENOLPHTHALEIN, PHENOLSULPHONPHTHALEIN, AND SOME HALOGEN DERIVATIVES.
}

By H. E. Howe and K. S. Gibson.

T $N$ this paper are given the results of a quantitative study of the absorption spectra of phenolphthalein and its halogen and sulphon derivatives with particular reference to the effect of the substitution of halogen for hydrogen and to the relation between chemical constitution and absorption in cases where color results from the addition of alkali to neutral solutions of the phthaleins.

The investigation was carried out in the physical laboratory of Cornell University, having been made possible by a grant from the Carnegie Institution to Professor E. L. Nichols. For information and suggestions concerning the chemistry of the problem the authors have been dependent upon Professor W. R. Orndorff and Dr. S. A. Mahood, of the department of chemistry, who have supplied the substances studied and have followed the work closely.

\section{The Compounds Studied.}

Phenolphthalein may be considered the parent substance from which the other compounds studied are derived by the substitution of chlorine, bromine, or iodine atoms for the hydrogen atoms attached to the carbon atoms of the benzene rings of the molecule. This compound, which is a condensation product of phthalic an-

hydride, $\mathrm{OC}-\mathrm{C}_{6} \mathrm{H}_{4}-\mathrm{CO}$ with phenol,

$\mathrm{C}_{6} \mathrm{H}_{5} . \mathrm{OH}$, is given the structural formula shown in Fig. I $(a)$. Phenoltetrachlorophthalein is a derivative in which four chlorine atoms are substituted for the hydrogen atoms in the phthalic acid residue, $i . e$. , for the four hydrogen atoms which are in the lower benzene ring in
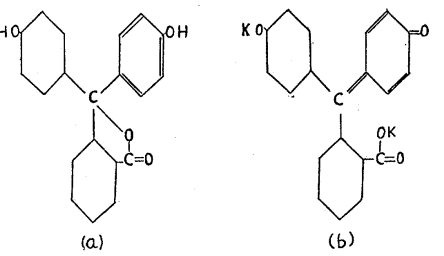

Fig. 1.

(a) Phenolphthalein.

(b) Di-potassium salt of phenolphthalein. the formula.

Both of the above compounds may have derivatives formed by the substitution of two bromine atoms or two iodine atoms in each of the 
benzene rings of the phenol part of the molecule. The resulting compounds are named tetrabromo- and tetraiodophenolphthalein and -phenoltetrachlorophthalein. The six substances are colorless in the finely divided crystalline form in which they are obtained. They do not dissolve in alkali-free water, but dissolve in neutral alcohol, forming colorless solutions. The addition of alkali to the neutral solutions causes the appearance of color.

The appearance of color in such cases has generally been assumed to be "accompanied by the transformation into a derivative of quinone," 1 and the graphic formula given to the di-potassium salt is that shown in Fig. I (b).

\section{Apparatus and Procedure.}

The absorption in the visible part of the spectrum was measured with a Lummer-Brodhun spectrophotometer, ${ }^{2}$ with the acetylene flame as a source. The ultraviolet absorption was determined photographically by means of a Hilger sector photometer ${ }^{3}$ in connection with a large Hilger quartz spectrograph. The source in this case was the aluminum spark under water, which gives a continuous spectrum as far as the quartz system will transmit.

The absorption curves are plotted to show the molecular absorption constant as a function of the frequency. The frequency is the reciprocal of the wave-length in millimeters, e. g., wave-length 5,00o A.U. equals frequency 2,000. The absorption constant $\beta$ is defined by the equations

$$
\frac{I}{I^{\prime}}=\mathrm{IO}^{\beta c d} \quad \text { or } \quad \beta=\frac{\mathrm{I}}{c d} \times \log _{10} \frac{I}{I^{\prime}}
$$

where $I$ represents the intensity of light transmitted by a cell filled with the pure solvent, $I^{\prime}$ the intensity of light transmitted by a similar cell filled with the solution, $d$ the thickness of the absorbing layer in centimeters, and $c$ the concentration of the solution. As a concentration of .ooor gram-molecule per liter, $i$. e., .ooor $N$, was found convenient for the photographic work, this concentration was taken as the unit. If the concentration were expressed in gram-molecules per liter, the constant $\beta$ obtained would be ro,ooo times that plotted. While a concentration of .000 I $N$ and a thickness of $\mathrm{I} \mathrm{cm}$. was satisfactory for most of the photographic work, the weak color of certain solutions made it necessary to vary the concentrations from $N /$ Ioooo to $N / 64$ and the thickness of the absorbing layer from $.3 \mathrm{~cm}$. to ro $\mathrm{cm}$.

A check on the accuracy of the measurements is given by the over-

${ }^{1}$ Perkin and Kipping, "Organic Chemistry," p. 53I.

${ }^{2}$ K. S. Gibson, Physical Review, 7, p. I94, I9i6.

${ }^{3}$ H. E. Howe, Physical Review, 8, p. 674, igi6. 
lapping of the curves obtained visually and photographically. The visual readings were extended in the blue nearly to frequency 2,200 , and the photographic measurements could be made to a frequency approximately 2,000 . The agreement of the two methods was very good in most cases.

The solutions were prepared in the following manner. A weighed amount of phthalein was dissolved in neutral absolute alcohol, the amount of alcohol being so chosen that a stock solution was obtained somewhat stronger than was desired for study. Neutral solutions for study were made by further diluting this stock solution, while alkaline solutions were prepared by adding to a measured volume of the stock solution a calculated volume of $N /$ roo solution of potassium hydroxide in absolute alcohol until there were present one, two, four, or ten molecules of alkali for each molecule of phthalein, after which more alcohol was added to obtain the desired concentration of phthalein.

Aquerous solutions cannot be so prepared, as the phthaleins will not dissolve in pure water. A weighed amount of phthalein was dissolved in a calculated volume of $N / \mathrm{IoO}$ potassium hydroxide in water, and more water was added to obtain the desired concentration of phthalein.

\section{Detalls of Absorption Spectra.}

\section{The Effect of the Substitution of Halogens.}

The neutral .0oor $N$ alcoholic solutions of the six phthaleins studied give absorption curves shown in Fig. 2. All six curves show more or less plainly two bands near frequency 3,500, and increasing general absorption

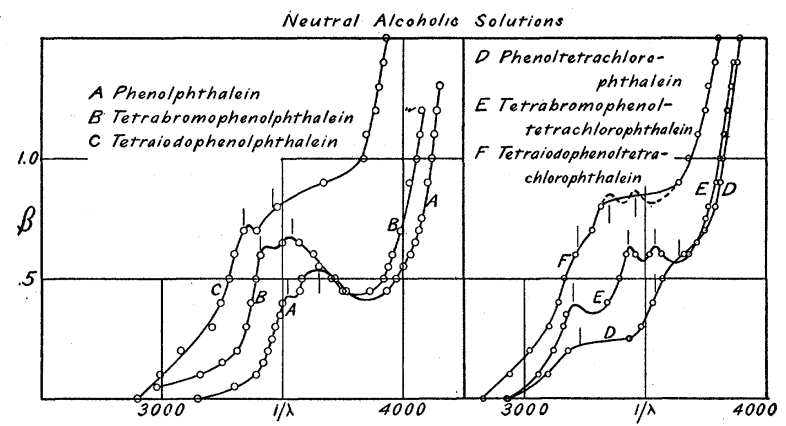

Fig. 2.

beyond 3,80o. Weaker solutions showed no other bands with frequencies less than 4,200, the working limit of the apparatus.

The short vertical lines indicate the positions of band centers as estimated directly from the negatives. When a negative is viewed as a whole the contrast effects of ten make the bands seem plainer than the 
curves show them, since the points plotted are found by examining a very small portion of the plate at a time. For example, the tetraiodophenoltetrachlorophthalein negative showed indications of two bands when viewed as a whole, while the actual values obtained gave a curve with merely a broad shoulder. The probable shape of the curve is indicated by a dotted line. The use of plate contrast to locate bands would not be allowable if the source gave other than a continuous spectrum.

The substitution of bromine or iodine for hydrogen increases the absorption and shifts the bands of lower frequencies, apparently without any change of frequency difference. The presence of four chlorine atoms in the phthalic acid residue, besides adding a shoulder to the side of the curve, seems to be accompanied by a coming together of the two bands. As seen on the curves, this approach seems to be effected by the shift of the band of lower frequency toward higher frequencies, and it is interesting to note that for alkaline solutions a similar shift of the ultra-violet bands toward higher frequencies accompanies the substitution of chlorine (Table I.).

TABLE I.

Frequencies of Absorption Bands for Alkaline Solutions. Alcoholic Solutions.

\begin{tabular}{|c|c|c|c|}
\hline \multirow{7}{*}{ 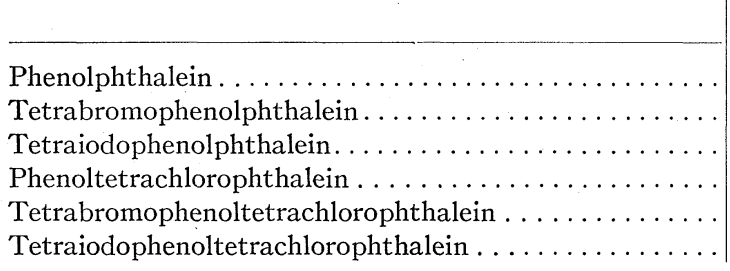 } & \multicolumn{3}{|c|}{ Band Frequencies. } \\
\hline & 1,780 & $2,750^{1}$ & $\ldots$ \\
\hline & 1,700 & $\ldots$ & 3,210 \\
\hline & 1,675 & $\ldots$ & 3,195 \\
\hline & 1,715 & $2,750^{1}$ & 3,570 \\
\hline & 1,625 & $\ldots$ & 3,265 \\
\hline & 1,600 & $\ldots$ & 3,250 \\
\hline \multicolumn{4}{|l|}{ Aqueous Solutions. } \\
\hline Phenolphthalein ......... & 1,810 & 2,710 & $3,500^{1}$ \\
\hline Tetrabromophenolphthalein... & 1,725 & $2,550^{1}$ & 3,280 \\
\hline Tetraiodophenolphthalein................. & 1,685 & $2,500^{1}$ & 3,200 \\
\hline Phenoltetrachlorophthalein $\ldots \ldots \ldots \ldots \ldots \ldots \ldots$ & 1,740 & 2,760 & 3,590 \\
\hline Tetrabromophenoltetrachlorophthalein ........... & 1,640 & $2,650^{1}$ & 3,290 \\
\hline Tetraiodophenoltetrachlorophthalein ........... & 1,610 & 2,570 & 3,240 \\
\hline
\end{tabular}

The change from neutral to alkaline solution is accompanied by a marked change in the character of the absorption (Figs. 3 and 4), which now shows a band in the visible and a single band near 3,200, followed by increasing general absorption beyond 3,500 . The following deviations

\footnotetext{
${ }^{1}$ Frequency of band center only approximate.
} 
from this general result may be cited: phenolphthalein with ten molecules of potassium hydroxide shows a weak band near 2,700 and a very slight
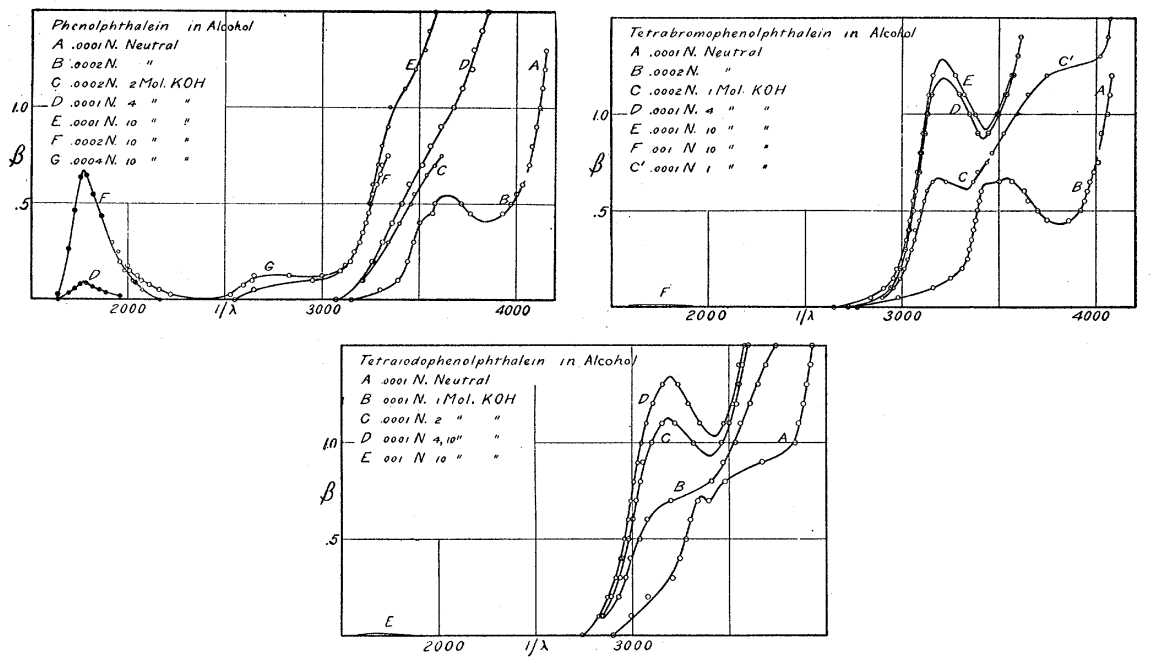

Fig. 3.

indication of a band near 3,400 , while phenoltetrachlorophthalein with six or ten molecules of alkali shows bands near 2,700 and 3,500. For the exact location of the bands, see Table I.
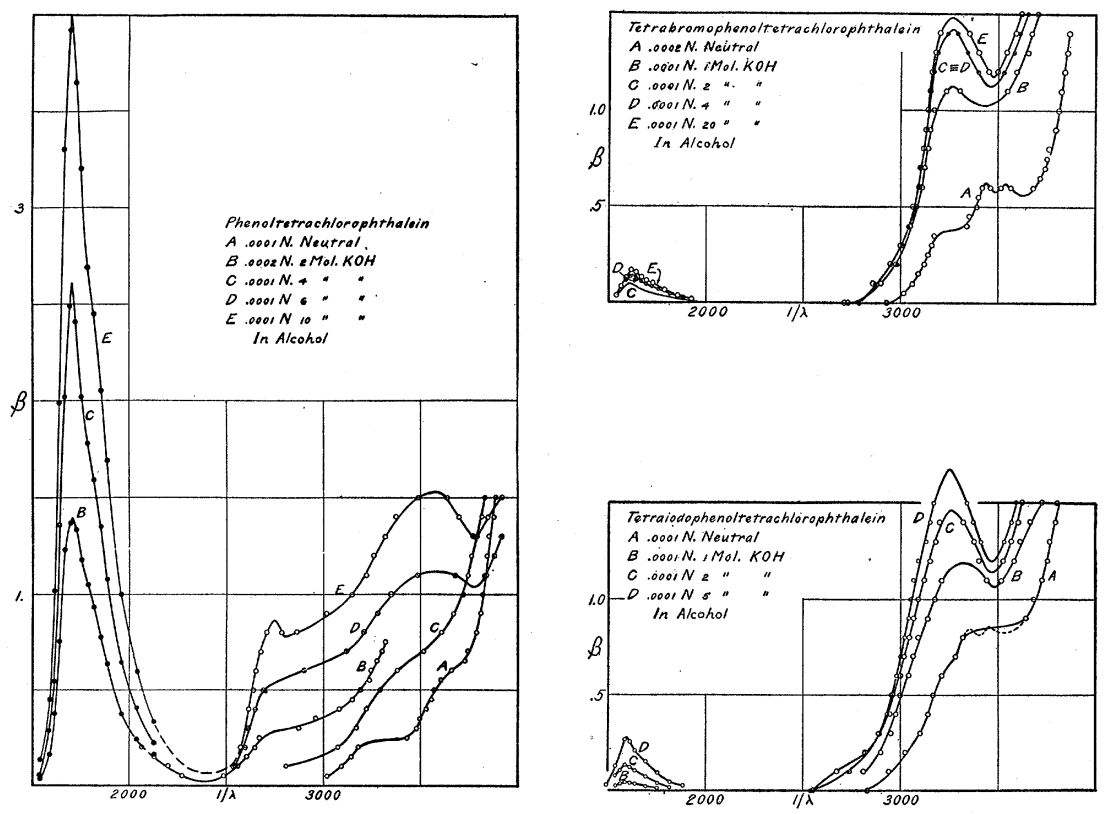

Fig. 4. 
Both visible and ultra-violet absorptions increase with the amount of alkali present up to twenty molecules, though this increase is not so rapid after the total amount of potassium hydroxide is more than two molecules per molecule of phthalein.

The band in the visible shifts toward the red with the substitution of chlorine, bromine, or iodine for hydrogen (Fig. 8).

The dilute solutions of tetrabromophenolphthalein and tetraiodophenolphthalein used for photographing did not show color through a I $\mathrm{cm}$. layer. In order to obtain the curves of absorption in the visible region it was necessary to use stronger solutions. It may be noted that the ultra-violet absorption of the apparently colorless solutions is of the same type as that of the colored solutions of the related compounds.

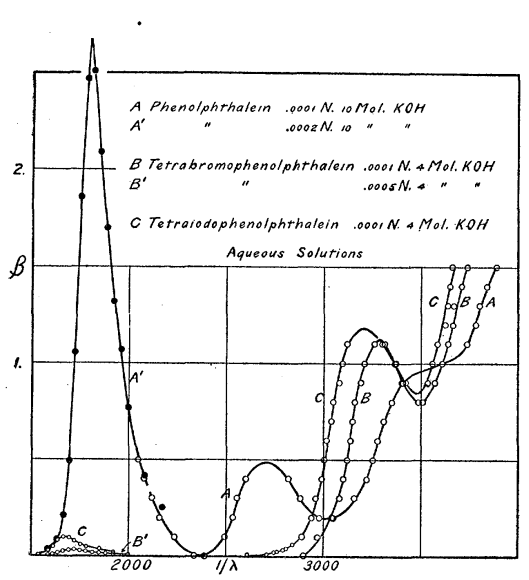

Fig. 5.

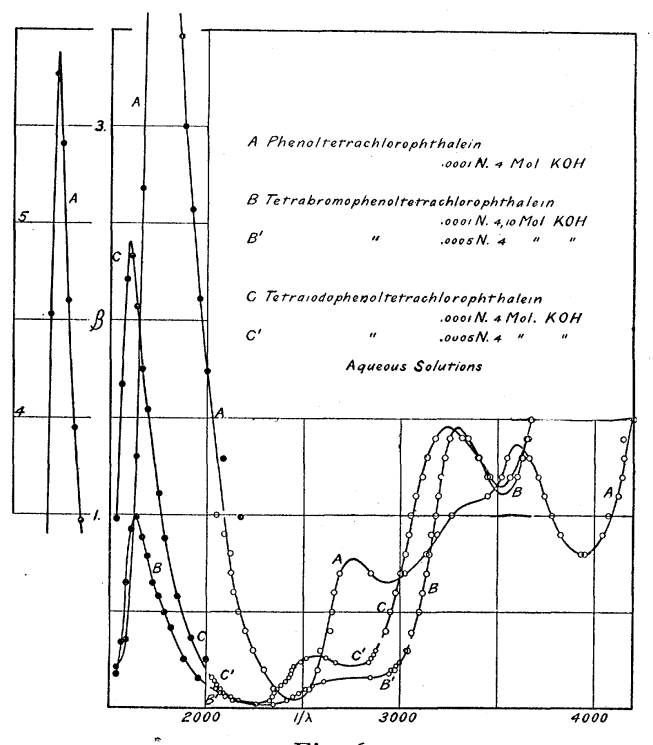

Fig. 6.

In the visible region the absorption bands of the aqueous solutions - (Figs. 5 and 6) are similar to those of the alcoholic solutions but the value of the maximum absorption is increased two to nine times and the bands are shifted toward higher frequencies. The solutions show increasing absorptions in the same order as do the alcoholic solutions of the same strength of alkali, viz., tetrabromophenolphthalein, tetraiodophenolphthalein, tetrabromophenoltetrachlorophthalein, tetraiodophenoltetrachlorophthalein, phenolphthalein, and phenoltetrachlorophthalein. The last-named substance has a maximum absorption I80 times the first in aqueous solution and $45^{\circ}$ times in alcoholic solution.

All of the aqueous solutions show weak ultra-violet bands near fre- 
quency 2,60o. To bring out this band photographs were made with stronger solutions and the points so obtained are indicated on the curves by small circles close together. The solutions of tetrabromo- and tetraiodophenolphthalein containing potassium hydroxide did not show this band with the low concentrations first used. As such a band had been earlier reported by Meyer and Fischer, ${ }^{1}$ solutions were prepared by their method of treating an excess of phthalein with half normal sodium hydroxide, filtering off the undissolved phthalein, and diluting the solution to the concentration indicated in Fig. 7, which shows the band in question. The value of the absorption constant here shown is not strictly comparable with that plotted in the other cases because of the different method of preparing the solution.

The maximum absorption in this weak band changes with the phthalein in the same order as does the band in the visible. This indicates a connection between the bands and suggests that a

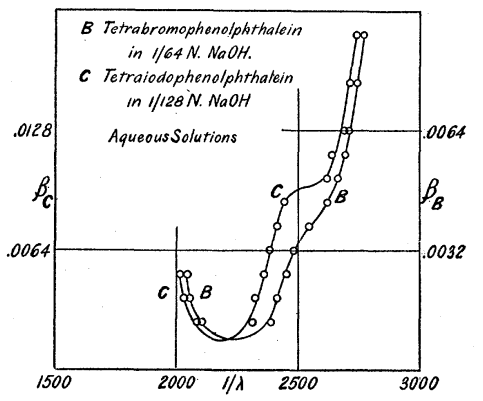

Fig. 7. corresponding band in the ultra-violet should appear in the alkaline alcoholic solutions. Such a band does appear in the solutions that are most strongly absorbing, $i$. e., phenolphthalein and phenoltetrachlorophthalein. ${ }^{2}$

This band, like the band in the visible, shifts toward lower frequencies with the substitution of bromine or iodine. But unlike the band in the visible, it shifts in the opposite direction upon the substitution of chlorine. A shift of this latter sort is shown by the stronger ultra-violet band near 3,200 when chlorine is substituted. This is true in both alcoholic and aqueous solutions. Hence in its shifts the weak ultra-violet band seems related to the other band in the ultra-violet, while in its variations in intensity it seems related to the band in the visible.

In striking contrast with the behavior of the bands in the visible and near 2,600, the prominent band near 3,200 in the ultra-violet shows a maximum absorption of approximately the same value for all solutions, alcoholic and aqueous. The absorption is slightly increased by the substitution of chlorine.

Fig. 8 shows the collected curves of visible absorption of the alkaline

1 Ber. d. Deut. Chem. Gesell., 44, p. I944, I9r I.

2 Since this paper was completed a careful examination of alkaline alcoholic solutions of tetrabromo- and tetraiodo-phenoltetrachlorophthalein of greatly varying concentrations has been made. No trace of the band near frequency 2,600 was found. 
solutions. The solutions used for obtaining the curves in the upper row contained ten molecules of potassium hydroxide per molecule of phthalein. Tetrabromo- and tetraiodophenolphthalein solutions were so weakly colored that the only solutions examined in the visible were $N /$ IOoo with ten molecules of alkali. The other solutions whose absorp-

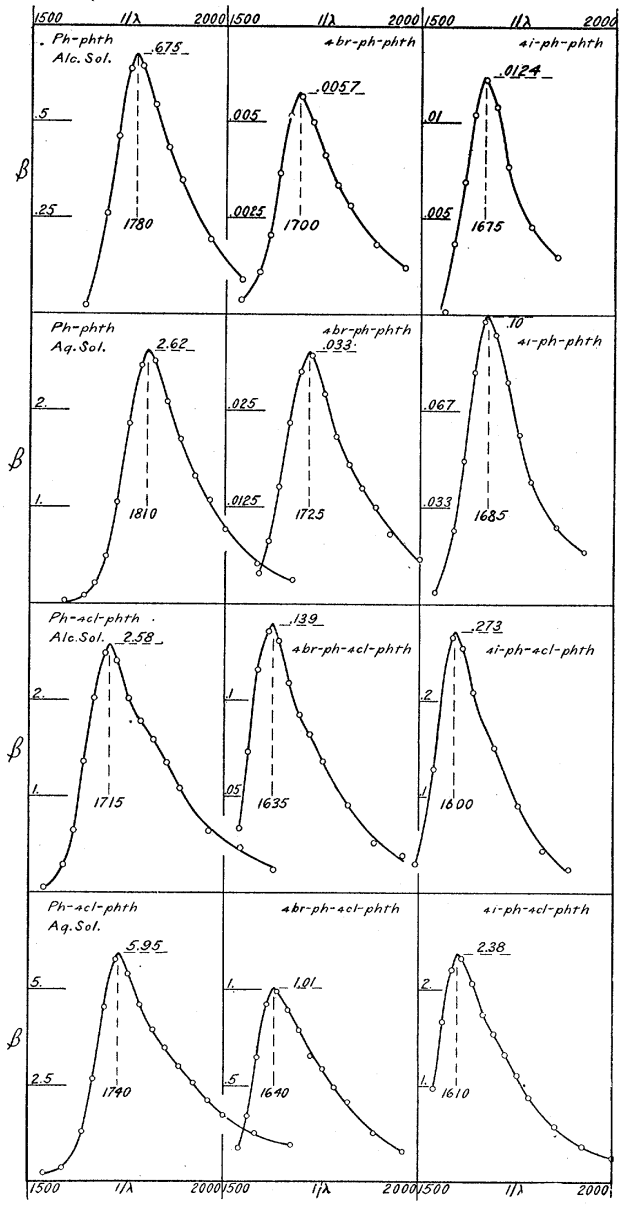

Fig. 8. tions are shown in Fig. 8 contained four molecules of alkali. While the shifts toward the red in the visible region are seen from Table I. and Fig. 8 to follow the general law of increase with increasing mass of substituent, it is also to be seen that the frequency of band center is not a function of molecular weight alone. Thus the salt of tetraiodophenolphthalein (mol. wt. 898) has a band centering at a frequency higher than that of tetrabromophenoltetrach lorophthalein (mol. wt. 848). The position of the absorption band might reasonably be expected to depend upon both the halogen substituted and upon its position in the molecule. With this in mind, the frequencies of band centers were plotted against molecular weights (Fig. 9) when it was found that the points lay on two curves corresponding to the two groups into which the compounds may be divided. One group includes phenolphthalein and those of its derivatives in which bromine or iodine are substituted in the phenol part of the molecule. The other group contains phenoltetrachlorophthalein and its corresponding derivatives. The authors propose to predict from these curves the centers of absorption bands that would be found for solutions of phthaleins in which chlorine is substituted in the phenol part of the molecule or in which bromine or iodine is substituted in the phthalic acid residue. ${ }^{1}$ 
TABLE II.

Alkaline Aqueous Solutions.

\begin{tabular}{|c|c|c|c|c|}
\hline & \multirow{2}{*}{ Band.No. } & \multicolumn{2}{|c|}{ Previously Found. } & \multirow{2}{*}{$\begin{array}{c}\text { Found by Authors. } \\
r / \lambda .\end{array}$} \\
\hline & & $\lambda$. & $\mathbf{x} / \lambda$. & \\
\hline Phenolphthalein. ........... & 1 & $\begin{array}{l}.5585^{1} \\
.5500^{2}\end{array}$ & $\begin{array}{l}1,790 \\
1,820\end{array}$ & 1,810 \\
\hline & $\begin{array}{l}2 \\
3\end{array}$ & $.372^{2}$ & 2,690 & $\begin{array}{l}2,710 \\
3,500 \text { (approx.) }\end{array}$ \\
\hline Tetrabromophenolphthalein........ & $\begin{array}{l}1 \\
2 \\
3\end{array}$ & $\begin{array}{l}.581^{1} \\
.5835^{2} \\
.389^{2}\end{array}$ & $\begin{array}{l}1,720 \\
1,715 \\
2,570\end{array}$ & $\begin{array}{l}1,725 \\
2,550 \text { (approx.) } \\
3,285\end{array}$ \\
\hline
\end{tabular}

${ }^{1}$ Meyer and Marx, Ber. d. Deut. Chem. Gesell., 4I, p. 2446, r9o8.

2 Meyer and Fischer, Ber. d. Deut. Chem. Gesell., 44, p. I944, I9II.

Table II. gives a comparison of the frequencies of the absorption bands for alkaline aqueous solution s as taken from the curves prevously published and as found by the authors. The failure of Meyer and Fischer to detect band No. 3 was probably due, in the case of phenolphthalein, to the fact that the band is a broad faint one superposed upon the increasing general absorption, and in the case of tetrabromophenolphthalein, to their failure to carry the concentrations over a great enough range. In this latter substance, band No. 2 was found by the authors not to be so pronounced (Fig. 7)

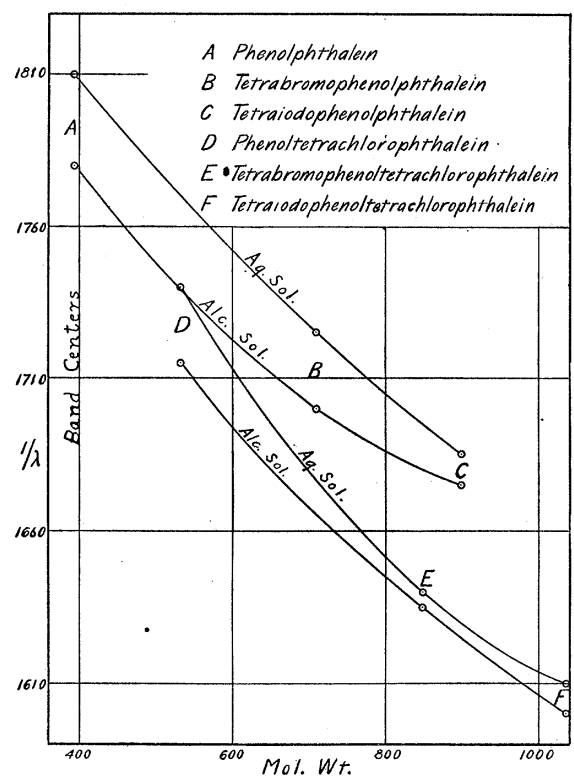

Fig. 9. as the curve given by Meyer and Fischer would indicate.

${ }^{1}$ At the time this prediction was proposed, phthaleins with the suggested halogen substitutions had not been prepared. Since this paper was completed, tetrachlorophenolphthalein has been prepared under the direction of Dr. S. A. Mahood, of the Department of Chemistry, and its absorption band determined by Mr. E. P. Tyndall, who found that alkaline solutions gave bands centering at 1695 in alcoholic solution and at 1,720 in aqueous solution. (See the following article.) Since the molecular weight of the di-potassium salt of the compound is 532, the frequencies predicted from the curves are 1,739 and 1,770 , and are far from agreement with those actually found. A further test of the prediction would be interesting. 


\section{The Relation of Absorption to Constitution.}

The difference in type of absorption spectra of solutions of a phthalein and of its di-potassium salt may be taken to mean that there is a fundamental difference in the structure of the two molecules. One way of representing this structural difference is shown in the graphic formulas given in Fig. I for phenolphthalein.

If all the alkali added to a solution of the phthalein reacted to form the colored salt, the conversion of the phthalein would be complete when there had been added two molecules of potassium hydroxide per molecule of phthalein, and the addition of more alkali would produce no effect on the absorption. The curves for the alcoholic solutions show that such a complete conversion of the phthalein does not take place. For example, the visible absorption of phenolphthalein could not be measured when only two molecules of alkali were present, and it continued to increase with alkali up to the greatest amount added, $i$. e., ten molecules. Further, when colored alcoholic solutions of phenolphthalein and of phenoltetrachlorophthalein containing two molecules of alkali were diluted with alcohol the color disappeared and the absorption in the ultra-violet reverted to the neutral type. This means that the excess of alcohol decomposes the salt, setting the phthalein free. The potassium ethylate resulting from the combination of the alcohol with the potassium of the colored salt has no absorption. Hence the solution gives the same absorption as the neutral solution of the phthalein.

The absorptions of solutions containing different relative amounts of phthalein and alkali were measured for the purpose of obtaining information concerning the compounds formed in the different cases. The curves of ultra-violet absorption of solutions with one molecule of alkali (Figs. 3 and 4) show that a partial conversion of the phthalein has taken place. The solutions showed a very slight color, with one molecule of alkali. In the visible region the absorption merely increases without change of type after the first appearance of color.

However, with a related compound, viz., phenolsulphonphthalein, it was possible to follow the gradual transition in type of spectrum in both visible and ultra-violet as the amount of alkali was increased. Fig. Io gives curves for the neutral solution and for solutions containing I, I $1 \frac{1}{2}$, and 2 molecules of alkali. The graphic formula is given, showing the quinoid structure assigned to this compound because it is colored. The neutral solution is yellow, the alkaline solution red. The change in color is progressive, due to the growth of a band in the yellow-green. The absorption of the alkaline aqueous solution (bands centering at $\mathrm{I}, 785,2,77 \mathrm{O}$, and 3,500 ) differs greatly in type from that of the neutral solution (bands at 2,320 and 3,770). 
Fig. II shows a similar change for alcoholic solutions. A rough examination of the absorption of tetrabromophenolsulphonphthalein indicated similar changes also. The physical evidence for a change in structure is as good in the case of these substances as in that of the

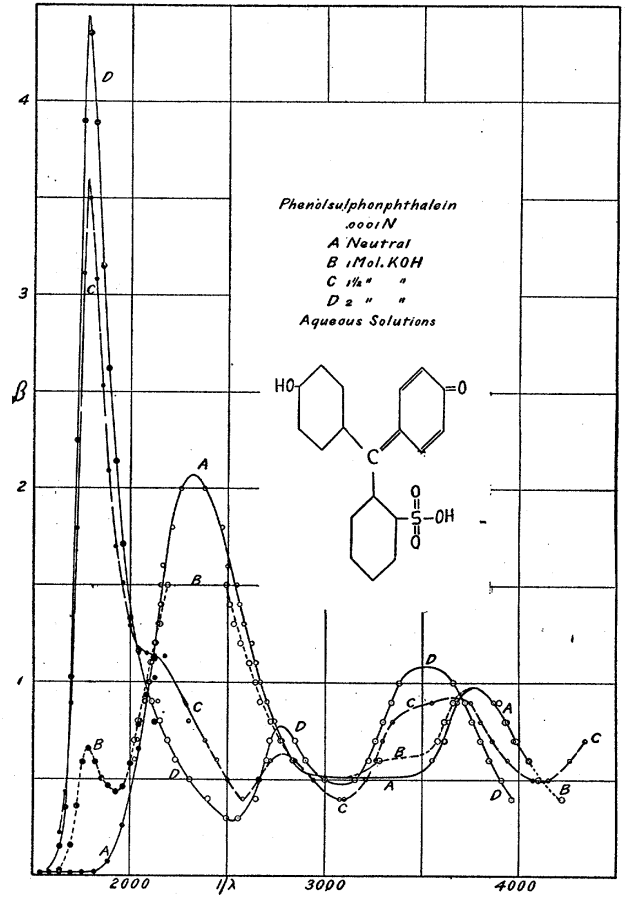

Fig. 10.

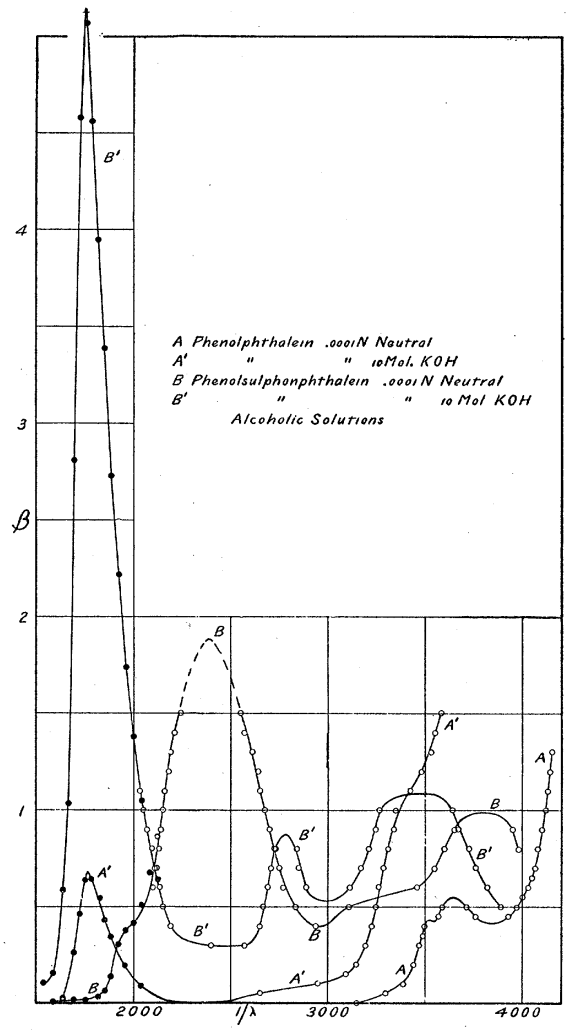

Fig. 11.

other phenolphthaleins discussed. The bearing of the facts here presented on chemical theory will be discussed in a later paper by Prof. W. R. Orndorff. It is hoped to continue the collection of data on absorption and constitution.

\section{SUMMARY.}

From a study of Table I. and the curves the following summarized statement of facts can be made.

Neutral alcoholic solutions of the phthaleins studied have absorption spectra of the same type (Fig. 2).

The type of absorption changes when the solutions become alkaline (Figs. 3,4 ). In some cases the change can be followed through a transition stage. 
The absorption of the aqueous solutions is of the same type as that of the alcoholic (Figs. 5, 6). The characteristic spectrum of the alkaline solutions consists of three absorption bands, one in the visible in the region $\mathrm{I}, 600-\mathrm{I}, 800$, the other two in the ultra-violet in the regions $2,500-2,700$ and $3,200-3,600$.

The maximum value of the absorption constant in the visible and in the band near 2,60o varies greatly with the phthalein, and is considerably greater in aqueous than in alcoholic solutions.

Band centers have a lower frequency for alcoholic solutions than for aqueous (Figs. 3, 4, 5, 6, 8).

The band in the visible region is shifted toward lower frequencies by the substitution of bromine or iodine in the phenol part of the molecule and of chlorine in the phthalic acid part of the molecule. The shift increases with the mass of the substituent and is less in alcohol than in water. Chlorine adds a shoulder to this band on the side toward higher frequencies (Fig. 8).

The band near 2,600 is shifted in the same direction as is the visible band by bromine and iodine, but is shifted toward higher frequencies by the chlorine, the shifts increasing with the mass of the substituent.

The band near 3,200 is also shifted toward lower frequencies by bromine and iodine and toward higher frequencies by chlorine. As in the case of the other bands, the shift by iodine is greater than by bromine.

It is hoped that the accumulation of further data may make possible general conclusions as to the relation of absorption to constitution of the phthaleins.

Cornell University,

August, 19I7. 